NASA Contractor Report 181986

ICASE Report No. 90-12

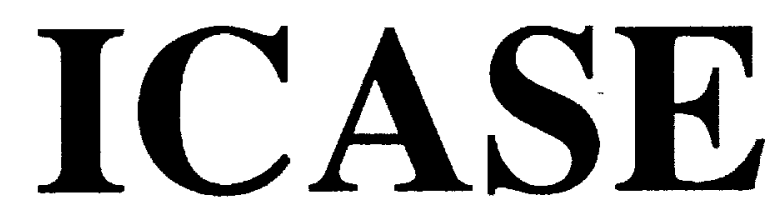

\title{
ROBUSTNESS OF CONTROLLERS DESIGNED USING GALERKIN TYPE APPROXIMATIONS
}

K. A. Morris

Contract No. NAS1-18605

January 1990

Institute for Computer Applications in Science and Engineering NASA Langley Research Center

Hampton, Virginia 23665-5225

Operated by the Universities Space Research Association

\section{N/SA}

National Aeronautics and

Space Administration

Langley Research Center

Hampton, Virginia 23665-5225

(NASA-CR-1OI9OS) ROSUSTNESS OF CONTROLLERS

DESIGNEO USINS GALERKIN TYPE APPROXIMATIONS

$N 90-19063$

Final Report (ICASE) ?1 P CSCL $20 \mathrm{~K}$ 


\title{
ROBUSTNESS OF CONTROLLERS DESIGNED USING GALERKIN TYPE APPROXIMATIONS
}

\author{
K. A. Morris ${ }^{1}$ \\ Institute for Computer Applications in Science and Engineering \\ NASA Langley Research Center \\ Hampton, VA 23665
}

\begin{abstract}
One of the difficulties in designing controllers for infinite-dimensional systems arises from attempting to calculate a state for the system. In this paper it is shown that Galerkin type approximations can be used to design controllers which will perform as designed when implemented on the original infinite-dimensional system. No assumptions, other than those typically employed in numerical analysis, are made on the approximating scheme.
\end{abstract}

\footnotetext{
${ }^{1}$ Part of this research was done while the author was with the Department of Electrical Engineering, University of Waterloo, Waterloo, Ontario, N2L 3G1. This research was also supported by the National Aeronautics and Space Administration under NASA Contract No. NAS1-18605 while the author was in residence at the Institute for Computer Applications in Science and Engineering (ICASE), NASA Langley Research Center, Hampton, VA 23665.
} 



\section{Introduction}

There are computational difficulties, apart from the theoretical problems, to designing controllers for a class of systems whose dynamics are described by partial differential equations. Unlike finite-dimensional linear dynamical systems, which are often easily solved for the state of the system, a closed form solution for the state of an infinite-dimensional system can be computed only in the simplest of situations. This is due to the difficulty of solving a partial differential equation. In general, it is necessary to use a numerical approximation to the state of the system when designing a controller.

This leads immediately to several questions regarding robustness. Is the use of finitedimensional approximations a valid technique when designing controllers for infinite-dimensional systems? If so, which approximation methods can be used to design controllers which will perform as designed when implemented on the actual system, and how high an order is required?

A wide class of projection methods, which includes mode truncation and finite element Galerkin techniques, is considered in this paper. It is shown that, if the order of an approximation to a system is sufficiently high, then the approximating system is stabilizable, and a controller designed using the approximation will stabilize the original system. Furthermore, the closed loop response of the system will be close to the the closed loop response of the approximating system. This result is independent of the technique used for controller design.

Other researchers have studied convergence of solutions to the Riccati equation [eg., 9, $10,12]$, but have assumed either uniform stability or stabilizability of the approximating systems. Balas $[1,2]$ assumes convergence of a stabilizing feedback control. However, the assumptions on an approximation scheme required for the results in this paper to hold are not stronger than those typically imposed on any numerical technique used in computer analysis. The key assumption is that the original infinite-dimensional system is stabilizable.

We consider semigroup control systems of the following form:

$$
\begin{gathered}
\dot{x}(t)=A x(t)+B u(t), \\
y(t)=C x(t), \\
x(0)=x_{0} \quad x_{0} \in \mathrm{D}(A) \subset X .
\end{gathered}
$$

$A$ satisfies the Hille-Yosida theorem on the Hilbert space $X$ and hence generates a strongly continuous $\left(C_{0}\right)$-semigroup $T(t)$ on $X$. Control and observation are assumed to be bounded i.e.,

$$
B \in \mathbf{B}\left(R^{m}, X\right) \text { and } C \in \mathbf{B}\left(X, R^{p}\right)
$$


This formulation includes a wide class of systems; in particular many problems concerning vibrations in structures with point sensing and actuation can be placed within this framework.

The inner product on $X$ is indicated by $($,$) and the corresponding norm by, \|\|$. Inner products on different spaces are not distinguished as the context indicates which space is meant. We use the following definition of internal stability.

Definition 1.1: The $C_{0}$-semigroup $T(t)$ is $\sigma$-stable if there exist constants $M$ and $\alpha>\sigma$ such that $\|T(t)\| \leq M e^{-\alpha t}$ for all $t \geq 0$.

The possibility of $\sigma>0$ is included to allow for situations where the purpose of controller design is for purposes other than simple stability (i.e., $\sigma=0$ ). For instance, while vibrating structures are generally open-loop stable, the settling time of these systems is often unacceptably large.

Several definitions will be required.

Definition 1.2: The pair $(A, B)$ is $\sigma$-stabilizable if there exists a bounded linear operator $K: X \rightarrow R^{m}$ such that $A-B K$ generates a $\sigma$-stable semigroup.

Definition 1.3: The pair $(A, C)$ is $\sigma$-detectable if there exists a bounded linear operator $F: R^{p} \rightarrow X$ such that $A-F C$ generates a $\sigma$-stable semigroup.

Definition 1.4: The system $(A, B, C)$ is jointly $\sigma$-stabilizable/detectable if $(A, B)$ is $\sigma$-stabilizable and $(A, C)$ is $\sigma$-detectable.

Define the linear space $\mathbf{A}$ to consist of functions of the form

$$
h=h_{a}(t)+\sum_{i=1}^{\infty} h_{i} \delta\left(t-t_{i}\right) .
$$

where $h_{a} \in L_{1}[0, \infty)$ and the sequence $\left\{h_{i}\right\}$ is absolutely summable. The norm of a function in $\mathbf{A}$ is

$$
\|h\|=\int_{0}^{\infty}\left|h_{a}(t)\right| d t+\sum_{i=0}^{\infty}\left|h_{i}\right|<\infty
$$

A SISO system is said to be $L_{\infty}$-stable or bounded-input bounded-output (BIBO) stable if and only if its impulse response belongs to the set $\mathbf{A}$. Moreover, the gain of an BIBO-stable system is equal to the norm of its impulse response in the set $\mathbf{A}$ [6]. The symbol $\hat{\mathbf{A}}$ is used to denote the set of Laplace transforms of distributions in $\mathbf{A}$.

Define $M(\mathbf{A})$ to be the set of matrices with elements in A. A MIMO system is $L_{\infty}$-stable if and only if its impulse response belongs to $M(\mathbf{A})$. The $L_{\infty}$-gain of a $m \times p$ matrix $H$ is 
the induced norm defined by

$$
\|H\|_{\mathbf{A}}:=\max _{i} \sum_{j=1}^{p}\left\|h_{i j}\right\|_{\mathbf{A}}
$$

Consider the common situation where the system is already stable and the aim of controller design is to improve its transient response. Let us specify a real number $\sigma>0$, which is the minimum acceptable stability margin. We define a system to be $\sigma-L_{\infty}$-stable if and only if its impulse response $f(t)$ is of the form (1.1), and instead of (1.2) satisfies the more stringent conditions

$$
\sum_{i=0}^{\infty}\left|h_{i}\right| \exp \left(\sigma t_{i}\right)<\infty, \int_{0}^{\infty}\left|h_{a}(t) \exp (\sigma t)\right| d t<\infty
$$

Let $\mathbf{A}_{\sigma}$ denote the set of all distributions satisfying, and let $\hat{\mathbf{A}}_{\sigma}$ denote the set of Laplace transforms of distributions in $\mathbf{A}_{\sigma}$.

We now define precisely what is meant by external stabilization of a system. Suppose $P$ is a given system, for which we wish to design a controller $C$, arranged in the familiar feedback configuration shown in Figure 1 . It is readily seen that the $2 \times 2$ transfer matrix $H(P, C)$ which maps the pair $\left(u_{1}, u_{2}\right)$ into the pair $\left(e_{1}, e_{2}\right)$ is given by

$$
H(P, C)=\left[\begin{array}{ll}
(I+P C)^{-1} & -P(I+C P)^{-1} \\
C(I+P C)^{-1} & (I+C P)^{-1}
\end{array}\right] .
$$

The feedback system, or alternatively the pair $(P, C)$, is said to be stable if each of the four elements in the above matrix belongs to the set $\mathbf{S}$ of stable transfer functions. Definition of $\mathbf{S}$ depends upon the application. Thus the closed loop system is BIBO- $\sigma$-stable if and only if all four elements belong to $\hat{\mathbf{A}}_{\sigma}$. It is important to note that the present definition of stability is symmetric in $P$ and $C$. Thus $P$ stabilizes $C$ if and only if $C$ stabilizes $P$.

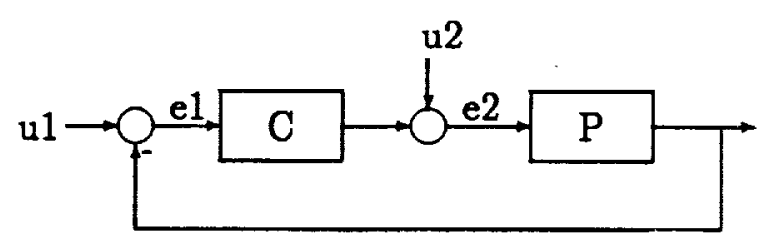

Figure 1. Feedback System 
The problem is to design a controller for the system (1.0) which achieves such objectives as external stability. Since this generally requires knowledge of the system state, the difficulty in solving partial differential equation systems and many other semigroup systems means that an approximation to the state must be used. In the next section we define a class of approximation techniques known variously as Galerkin and projection techniques.

\section{Approximation Technique}

Suppose we have a sequence of finite-dimensional subspaces $X_{h}$ with $X_{h_{1}} \subset X_{h_{2}}$ for $h_{1}>h_{2}$. We assume that $X_{h} \subset \mathbf{D}(A)$. When finite elements are used as a basis for the approximating subspace $X_{h}$, then $A$ should be understood in its weak or extended sense [7, 20]. Define $P_{h} x$ as the orthogonal projection of $x \in X$ onto the finite-dimensional subspace $X_{h}$. The parameter $h$ converges to zero as the order of the approximation is increased. Since all norms on $X_{h}$ are equivalent, we will assume that the norm on $X_{h}$ is that inherited from $X$. Since $P_{h}$ is an orthogonal projection, it follows that

$$
\left\|P_{h} x\right\| \leq\|x\|
$$

The approximating system is $\left(A_{h}, B_{h}, C_{h}\right)$ where

$$
\begin{aligned}
B_{h} & :=P_{h} B \\
C_{h} & :=\left.C\right|_{X_{h}}
\end{aligned}
$$

and $A_{h}$ is the bounded linear operator defined by

$$
A_{h} x_{h}=P_{h} A x_{h} \text { for all } x_{h} \in X_{h}
$$

In common with [3], the operators $A_{h}, B_{h}, C_{h}$ and the semigroup $T_{h}(t)$ generated by $A_{h}$ are only defined on $X_{h}$.

We make the following assumptions:

A1) The projection operators $P_{h}$ converge strongly to the identity on the Hilbert space $X$. That is, for all $x \in X$

$$
\lim _{h \rightarrow 0}\left\|P_{h} x-x\right\|=0 .
$$

A2) The approximation scheme approximates the solution space not the operator $A$, in that $x \in X_{h}$ implies $A x \in X_{h}$ so that

$$
A_{h} x=A x \text { for } x \in X_{h} .
$$

Modal truncation and many applications of the finite element method satisfy this assumption. 
A3) A core $\mathbf{C}$ of a closed operator $A$ is a linear space contained in the domain of $A$ with the property that the set of elements $(x, A x), x \in \mathbf{C}$ is dense in the graph $\mathbf{G}(A)$ of the operator $A$ ([15] pg. 166). In other words, if we define an operator $L$ to be the restriction of $A$ to $\mathbf{C}$ so that

$$
\begin{gathered}
\mathrm{D}(L)=\mathbf{C} \\
L x=A x, \quad x \in \mathbf{C}
\end{gathered}
$$

then $A$ is the closure of $L$, or equivalently, $\overline{\mathrm{G}(L)}=\mathrm{G}(A)$.

We will assume that there exists a core $\mathbf{C}$ for $A$ such that

$$
\lim _{h \rightarrow 0}\left\|P_{h} A x-A_{h} P_{h} x\right\|=0, \text { for all } x \in \mathrm{C} .
$$

Such an approximation scheme is said to be consistent.

We will further assume that the semigroups $T_{h}(t)$ generated by $A_{h}$ are uniformly bounded, that is, there exist real numbers $H, M \geq 1$ and $\omega$ such that

$$
\left\|T_{h}(t)\right\| \leq M e^{\omega t} \text { for all } h \leq H .
$$

(Uniform boundedness of the approximate semigroups is generally referred to as "stability" in the numerical analysis literature.) Consistency and uniform boundedness are required for convergence of the approximation [e.g., 16, 18, 20] i.e., for all $\varepsilon>0, t>0$ and for all $x \in X$ there exists $H$ such that

$$
\left\|P_{h} T(\tau) x-T_{h}(\tau) P_{h} x\right\|<\varepsilon \text { for all } \tau \in[0, t] \text { and } h<H .
$$

Assumptions (A1)-(A3) are satisfied by typical approximation methods such as finite element techniques and modal truncation.

In subsequent sections the original semigroup control system will be assumed stabilizable and/or detectable. However, no assumptions about the stabilizability or detectability of the approximations are made. In the next section it is shown that stabilizability of the approximating systems can be deduced from stabilizability of the original semigroup control system.

\section{Stabilizability of Approximating Systems}

We require the following theorem which guarantees the existence of a solution to the infinite-dimensional Riccati equation if the pair $(A, B)$ is stabilizable.

Theorem 3.1: [17] Let $A$ and $B$ be as defined above and let $L, Q$ be self-adjoint and positive definite bounded linear operators. If there exists a solution $u^{*}$ to the optimal control 
problem with the quadratic cost function

$$
J(u):=\int_{0}^{\infty}[(L x(t), x(t))+(Q u(t), u(t))] d t
$$

which satisfies the condition that $J\left(u^{*}\right)$ be finite, then there exists an unique self-adjoint and non-negative solution $\Pi \in \mathrm{L}(X, X)$ to the infinite-dimensional Riccati equation

$$
A^{*} \Pi+\Pi A-\Pi B Q^{-1} B^{*} \Pi+L=\Theta
$$

where $\Theta$ is the zero operator. Let $K=Q^{-1} B^{*} \Pi$; then $A-B K$ generates an exponentially stable $C_{\mathrm{o}}$-semigroup.

Clearly an admissible solution exists if and only if $(A, B)$ is stabilizable.

Theorem 3.2: Assume we have a semigroup control system (2.0) and an approximation scheme satisfying assumptions (A1)-(A3) in Section 2. Assume that the system is stabilizable. Then, for sufficiently small $h$, the approximating systems are stabilizable i.e., there exists an operator $K_{h}$ such that $A_{h}-B_{h} K_{h}$ generates an exponentially stable semigroup on $X_{h}$.

Proof: For each approximating subspace, define a self-adjoint operator $\Pi_{h}: X_{h} \rightarrow X_{h}$ as

$$
\Pi_{h}:=P_{h} \Pi P_{h}
$$

where $\Pi$ is the solution to the Riccati equation (3.2). It will be shown that, for small enough $h, \Pi_{h}$ solves the finite-dimensional Riccati equation

$$
A_{h}^{*} \Pi_{h}+\Pi_{h} A_{h}-\Pi_{h} B_{h} Q^{-1} B_{h}^{*} \Pi_{h}+L_{h}=\Theta_{h}
$$

where $\Theta_{h}$ is the zero operator in $\mathbf{B}\left(X_{h}, X_{h}\right)$ and $L_{h}$ is a self-adjoint positive definite operator (as yet undetermined) in $\mathbf{B}\left(X_{h}, X_{h}\right)$.

Define the operator $R\left(L_{h}\right): X_{h} \rightarrow X_{h}$ by

$$
R\left(L_{h}\right)=A_{h}^{*} \Pi_{h}+\Pi_{h} A_{h}-\Pi_{h} B_{h} Q^{-1} B_{h}^{*} \Pi_{h}+L_{h}
$$

where $L_{h} \in \mathbf{B}\left(X_{h}, X_{h}\right)$ is as yet unspecified. Let us calculate a representation for $R\left(L_{h}\right)$ with respect to some basis $\left\{\phi_{i}\right\}_{i=1}^{n}$ for $X_{h}$. Using (3.2), we have

$$
\left(\phi_{i}, R\left(L_{h}\right) \phi_{j}\right)=\left(\phi_{i}, Q(h) \phi_{j}\right)+\left(\phi_{i}, L_{h} \phi_{j}\right)-\left(\phi_{i}, L \phi_{j}\right)
$$

where

$$
Q(h):=\Pi B Q^{-1} B^{*} \Pi-P_{h} \Pi P_{h} B Q^{-1} B^{*} P_{h} \Pi P_{h} .
$$


Now define $L_{h}: X_{h} \rightarrow X_{h}$ as

$$
L_{h} x_{h}=L x_{h}-Q(h) x_{h} \text { for all } x_{h} \in X_{h} .
$$

Then $R\left(L_{h}\right)=\Theta$ on $\mathbf{B}\left(X_{h}, X_{h}\right)$ since

$$
\left(\phi_{i}, R\left(L_{h}\right) \phi_{j}\right)=0 \quad \text { for all } \phi_{i}, \phi_{j} .
$$

In order to show that $\Pi_{h}$ solves a Riccati equation for $\left(A_{h}, B_{h}\right)$ for small enough $h$, it remains only to prove that $L_{h}$ is self-adjoint and positive definite for small $h$. Now, $\lim _{h \rightarrow 0}\left\|\left(I-P_{h}\right) B\right\|=0$ since $B$ operates on a finite-dimensional space. It follows that [22, Theorem 4.14]

$$
\lim _{h \rightarrow 0}\|Q(h)\|=\Theta .
$$

The fact that $L_{h}$ is self adjoint follows easily from the definition of $Q(h)$. Since $L$ is positive definite and self-adjoint we can choose $H$ so that for all $h<H$

$$
\left(x_{h}, L_{h} x_{h}\right) \geq c^{2}\left(x_{h}, x_{h}\right)
$$

for some $c>0$. Thus $L_{h}$ is self-adjoint and positive definite for all $h<H$, and $\Pi_{h}:=P_{h} \Pi P_{h}$ solves the Riccati equation for $\left(A_{h}, B_{h}\right)$ with weights $Q$ and $L_{h}$ if $h$ is sufficiently small. Defining $K_{h}:=Q^{-1} B_{h}^{*} \Pi_{h}, A_{h}-B_{h} K_{h}$ generates a stable semigroup on $X_{h}$ for $h<H$.

Banks and Kunisch [3] derived a similar result for parabolic systems using different assumptions. Rosen [19] considered the problem of convergence of solutions to approximating Riccati equations. However he considers a much smaller class of problems: his proof applies to cases where the open loop system is stable and $\Pi$ is a Hilbert-Schmidt operator. Although the result given here is weaker in that convergence of $\Pi_{h}$ to $\Pi$ in an operator norm is not shown, the theorem is considerably more general.

The following corollary is a consequence of the two results above.

Corollary 3.2a: Assume that $(A, B)$ is $\sigma$-stabilizable and let $\Pi$ be the solution to the Riccati equation for $(A+\sigma I, B)$ with weights $Q$ and $R$ as in equation (3.2). Define $\Pi_{h}:=P_{h} \Pi P_{h}$ and let $K_{h}=Q^{-1} B_{h}^{*} \Pi_{h}$. Then for sufficiently small $h, A_{h}-B_{h} K_{h}$ generates a $\sigma$-exponentially stable semigroup on $X_{h}$.

The results above for $\sigma$-stabilizable systems have obvious extensions to $\sigma$-detectable systems since $(A, C)$ is $\sigma$-detectable if and only if $\left(A^{*}, C^{*}\right)$ is $\sigma$-stabilizable.

Theorem 3.3: Let $(A, B, C)$ be a semigroup control system and suppose we have an approximation scheme which satisfies assumptions (A1)-(A3) of Section 2. Assume furthermore that $(A, B)$ is $\sigma$-stabilizable. Choose $\Pi \in \mathbf{B}(X, X)$ such that $\Pi$ solves the Riccati equation 
(3.2). Define $K:=Q^{-1} B^{*} \Pi$ and let $S(t)$ to be the semigroup generated by $A-B K$. Similarly, define $K_{h}:=Q^{-1} B_{h}^{*} P_{h} \Pi P_{h}$ and let $S_{h}(t)$ be the semigroup generated by $A_{h}-B_{h} K_{h}$. Then $S_{h}(t)$ converges to $S(t)$ uniformly on bounded intervals; i.e., for all $\epsilon>0, t>0$ and for all $x \in X$ there exists $H$ such that

$$
\left\|P_{h} S(\tau) x-S_{h}(\tau) P_{h} x\right\|<\epsilon \text { for all } \tau \in[0, t] \text { and } h<H .
$$

\section{Proof:}

$$
\left\|B_{h} K_{h}\right\| \leq\left\|B Q^{-1} B^{*}\right\|\|\Pi\|:=c .
$$

Using (2.2) and [18, Theorem 3.1.1] it follows that there exist real numbers $H, M \geq 1$ and $\omega$ such that

$$
\left\|S_{h}(t)\right\| \leq M e^{(\omega+M c) t} \text { for all } t \geq 0, \quad \text { for all } h \leq H .
$$

Define $A_{o}:=A-B K$ and $A_{h o}:=A_{h}-B_{h} K_{h}$. Let $\mathrm{C}$ be the core for $A$ such that the consistency condition (2.1) is satisfied. Since $B K$ is a bounded operator, $\mathbf{C}$ is also a core for $A_{\circ}$. The resolvent of $\left(\lambda I-A_{\circ}\right)$ is bounded for $\operatorname{Re}(\lambda)>-\sigma$, and so $\left(\lambda I-A_{\circ}\right) \mathrm{C}$ is dense in $X$ for $\operatorname{Re} \lambda>-\sigma$. It follows that for every $x \in \mathrm{C}$, we have

$$
\lim _{h \rightarrow 0}\left\|A_{h o} P_{h} x-P_{h} A_{o} x\right\| \leq \lim _{h \rightarrow 0}\left\|P_{h} B Q^{-1} B^{*}\left(P_{h} \Pi P_{h}-\Pi\right) x\right\|=0 .
$$

In order to show convergence of $\left\{S_{h}\right\}$ to $S$ it necessary to introduce a semigroup which is defined on all of $X$. Define $\tilde{A}_{h o}=A_{h o} P_{h}$. Since $\tilde{A}_{h o}$ is a bounded linear operator it generates the $C_{o}$-semigroup $\tilde{S}_{h}(t)$ defined by

$$
\tilde{S}_{h}(t)=\sum_{k=0}^{\infty} \frac{t^{k}}{k !} \tilde{A}_{h o}^{k}=\sum_{k=0}^{\infty} \frac{t^{k}}{k !} A_{h o}^{k} P_{h}=S_{h}(t) P_{h} .
$$

Since $\left\|\tilde{S}_{h}(t)\right\| \leq\left\|S_{h}(t)\right\|, \tilde{S}_{h}(t)$ also satisfies (3.3). Now, for $x \in \mathbf{C}$, we have

$$
\lim _{h \rightarrow 0}\left\|\tilde{A}_{h o} x-A x\right\| \leq \lim _{h \rightarrow 0}\left\|A_{h o} P_{h} x-P_{h} A_{o} x\right\|+\left\|P_{h} A_{o} x-A_{o} x\right\| .
$$

The first term was shown above to tend to zero. The second term converges to zero since $P_{h}$ converges strongly to the identity. It follows that

$$
\lim _{h \rightarrow 0} \sup _{0 \leq t \leq \tau}\left\|\tilde{S}_{h}(t) x-S(t) x\right\|=0 \quad \text { for all } x \in X \text {, for all } \tau \geq 0
$$

by the Trotter-Kato Approximation Theorem [18, Theorem 4.5]. Therefore, for all $x \in$ $X, \tau \geq 0$,

$$
\lim _{h \rightarrow 0} \sup _{0 \leq t \leq \tau}\left\|S_{h}(t) P_{h} x-P_{h} S(t) x\right\|=0
$$


The following theorem is now immediate.

Theorem 3.4: Suppose that the system $(A, B, C)$ is stabilizable and that assumptions (A1)-(A3) hold. Assume also that the approximation scheme also satisfies the additional condition (A4):

$$
\lim _{h \rightarrow 0} \sup _{0 \leq t \leq \tau}\left\|T_{h}^{*}(t) P_{h} x-P_{h} T^{*}(t) x\right\|=0 .
$$

Then for sufficiently small $h$, the Riccati equation

$$
A_{h}^{*} \pi_{h}+\pi_{h} A_{h}-\pi_{h} B_{h} Q^{-1} B_{h}^{*} \pi_{h}+P_{h} L P_{h}=\Theta_{h}
$$

has a unique non-negative self-adjoint solution $\pi_{h}$. The sequence of operators $\pi_{h}$ converge strongly to the solution to the exact equation (3.2). Define the optimal feedback operators $K_{h}:=R^{-1} B_{h}^{*} \Pi_{h}$ and $K:=R^{-1} B \Pi$; the semigroup generated by $A_{h}-B_{h} K_{h}$ converges to the semigroup generated by $A-B K$.

Proof: By assumption, hypotheses (H1)-(H2) in [12] are satisfied. Since Theorem 3.3 implies uniform stabilizability/detectability it follows that the sequence of solutions to the approximate Riccati equations are bounded by some real $M_{1}$ [12]. Since $\left\{\pi_{h}\right\} \in B(H, H)$ the sequence contains a weakly convergent subsequence. It is clear that the limit of this subsequence is also self-adjoint and non-negative. In fact, the limit is the solution to the exact Riccati equation, $\Pi$ [Theorem 6.7, 8]. A proof identical to that of [Theorem 6.9, 8] yields the remainder of the result.

The fourth assumption can easily be shown to hold for a wide class of problems. However, while assumptions (A1)-(A3) are almost always satisfied by a numerical scheme which is satisfactory for approximation purposes, (A4) may not be satisfied. This can lead to unsatisfactory controller design. An example of this situation is discussed in [4].

\section{Convergence in the Graph Topology}

The graph and gap topologies [22, 23] arose from a need to define convergence of possibly unbounded operators. The basic idea was outlined in Kato [15]. If $M, N$ are closed linear subspaces of a Banach space $X$ then $\delta(M, N)$ is the smallest number $\delta$ such that

$$
\delta \geq \operatorname{dist}(u, N)=\inf _{v \in N}\|u-v\| \quad \text { for all } u \in M .
$$

The gap between $M$ and $N$ is defined by

$$
\hat{\delta}(M, N):=\max (\delta(M, N), \delta(N, M)) .
$$


If $S$ and $T$ are closed operators from $X$ to $Y$ then the gap between their graphs as closed subspaces of $X \times Y$ is well defined. The difficulty in defining a gap for unstable systems is that the plant may not be represented by a mapping between two Banach spaces. However, some bounded inputs $u$, will be mapped to bounded outputs $y$. Let $\mathrm{U}$ be the set of stable inputs and outputs and let $P$ be the impulse response of a scalar plant. We will assume that $\mathbf{U}$ is a Banach space. The symbol " $*$ " denotes convolution. Define

$$
\mathbf{D}_{\mathbf{U}}:=\{u \in \mathbf{U} \text { such that } y \in \mathbf{U}\}
$$

and the operator

$$
P_{\mathrm{U}} * u:=P * u
$$

with domain $D_{\mathbf{U}}$. Then the graph of $P_{\mathrm{U}}$ is

$$
G\left(P_{\mathrm{U}}\right)=\left\{(u, y), y=P * u, u \in \mathrm{D}_{\mathrm{U}}\right\}
$$

It is easy to show that $G\left(P_{\mathrm{U}}\right)$ is closed if $P$ is a closed operator. The graph of a plant is now defined to be the set of bounded (in the appropriate sense) input-output pairs, i.e.,

$$
\mathrm{G}_{\mathrm{U}}(P)=G\left(P_{\mathrm{U}}\right)=\left\{(u, y) \in \mathbf{U}^{m+p}, y=P * u\right\} .
$$

In the case of $B I B O$ stability,

$$
\mathbf{G}_{\infty}(P)=\left\{(u, y) \in L_{\infty}^{m+p},\right\} .
$$

Define the gap between operators as

$$
d\left(P_{1}, P_{2}\right)=\hat{\delta}\left(\mathbf{G}_{\mathbf{U}}\left(P_{1}\right), \mathbf{G}_{\mathbf{U}}\left(P_{2}\right)\right) .
$$

With this metric the space of closed operators from $\mathbf{U}$ to $\mathbf{U}, \mathbf{C}(\mathbf{U}, \mathbf{U})$ becomes a metric space. Convergence of a sequence $P_{n} \rightarrow P$ is defined by $d\left(P_{n}, P\right) \rightarrow 0$. If a sequence of plants $\left\{P_{i}\right\}$ are stable, then convergence in norm to $P$ is equivalent to convergence in gap. The topology induced by the metric $d\left(P_{1}, P_{2}\right)$ is thus an appropriate generalization of the uniform topology to unstable plants.

Note that the above definition of "stable graphs" includes the graphs of non-causal plants. A more useful topology for our purposes, which considers only linear causal plants, can be obtained through the frequency domain approach of Vidyasagar [22]. Let the set of stable plants $\mathbf{S}$ be $\hat{\mathbf{A}}, P$ a plant with a right coprime factorization $(N, D)$ over $\hat{\mathbf{A}}$. Define $\hat{\mathbf{G}}_{\infty}(P)$ to be the Laplace transform of elements of $\mathrm{G}_{\infty}(P)$. Then

$$
\hat{\mathrm{G}}_{\infty}(P):=\left\{\left(\hat{u}, N D^{-1} \hat{u}\right), u \in L_{\infty}^{m}\right\}
$$




$$
\hat{\mathrm{G}}_{S}(P)=\left\{(D \hat{z}, N \hat{z}), z \in L_{\infty}^{m}\right\} \text {. }
$$

The graph topology for unstable plants is defined in [22] using right coprime factorizations (r.c.f.'s). Essentially, a basic neighborhood in the graph topology of a plant with a r.c.f. $\left(N_{o}, D_{0}\right)$ is the set of all plants with a r.c.f. $(N, D)$ where $N$ is close to $N_{0}$ in norm and $D$ is close to $D_{o}$. Define $\mathbf{S}$ to be the usual algebra of stable rational functions. For finitedimensional plants the graph topology is the topology induced on the quotient space $\mathbf{S} / \mathbf{S}$ by the set of stable functions, $\mathbf{S}$. Further details can be found in [22]. Zhu [24] presents a generalization of the graph and gap topologies to subsets of general quotient fields. Here, we consider only a particular set.

Callier and Desoer [5] define a quotient algebra of transfer functions of distributed systems which is useful for studying control of semigroup systems.

We say that a transfer function $f$ in $\hat{\mathbf{A}}$ is bounded away from zero at infinity if for every sequence $\left\{s_{k}\right\}$ with $\operatorname{Re}(s) \geq 0$ and

$$
\left|s_{k}\right| \rightarrow \infty
$$

we have

$$
\lim \inf _{k \rightarrow \infty}\left|h\left(s_{k}\right)\right|>0
$$

Define the sets

$$
\begin{gathered}
\hat{\mathbf{A}}_{-}=\left\{h \in \hat{\mathbf{A}}_{\alpha} \text { for some } \alpha>0\right\} \\
\hat{\mathbf{A}}_{-, \infty}=\left\{h \in \hat{\mathbf{A}}_{-}, f \text { bounded away from zero at infinity }\right\}
\end{gathered}
$$

and the corresponding quotient algebra

$$
\hat{\mathbf{B}}=\left\{h=a / b, a \in \hat{\mathbf{A}}_{-}, b \in \hat{\mathbf{A}}_{-, \infty}\right\}
$$

The assumption that $b \in \hat{\mathbf{A}}_{-, \infty}$ implies that functions in $\hat{\mathbf{B}}$ possess only a finite number of unstable poles. The extension to the multivariable case is straightforward, and we define $M(\hat{\mathbf{B}})$ to be the set of transfer function matrices with entries in $\hat{\mathbf{B}}$.

An extension which restricts the region of stability to $\operatorname{Re}(s)<-\sigma$ is also straightforward. The algebra $\hat{\mathbf{A}}$ is replaced by $\hat{\mathbf{A}}_{\sigma}$ in the above definitions and

$$
\hat{\mathbf{B}}_{\sigma}=\left\{h=a / b, a \in \hat{\mathbf{A}}_{\sigma_{1}-}, b \in \hat{A}_{\sigma, \infty}\right\} \text {. }
$$

Jacobson [13] and Jacobson and Nett [14] have shown that semigroup control systems which are $\sigma$-stabilizable and/or $\sigma$-detectable have transfer functions in $M\left(\hat{\mathrm{B}}_{\sigma}\right)$. This framework is thus appropriate for studying semigroup control systems using frequency domain methods. Furthermore, every function $P$ in $M\left(\hat{\mathbf{B}}_{\sigma}\right)$ has both a left- and a right-coprime 
factorization over $M\left(\hat{\mathbf{A}}_{\sigma}\right)$. Thus Vidyasagar's graph topology is well-defined for semigroup control systems providing they are $\sigma$-stabilizable and/or -detectable.

Theorem 4.1: Suppose $F_{b}$ is a sequence in $M\left(\hat{\mathbf{B}}_{\sigma}\right)$, and that $F \in M\left(\hat{\mathbf{B}}_{\sigma}\right)$. Then the following statements are equivalent.

(i) $\left\{F_{h}\right\}$ converges to $F$ in the graph topology.

(ii) There exist a r.c.f $(N, D)$ of $F$, and a sequence of r.c.f.'s $\left(N_{h}, D_{h}\right)$ of $F_{h}$ such that $N_{h} \rightarrow N$ and $D_{h} \rightarrow D$ in $M\left(\hat{\mathbf{A}}_{\sigma}\right)$

(iii) There exist a l.c.f. $(N, D)$ of $F$, and a sequence of l.c.f.'s $\left(N_{h}, D_{h}\right)$ of $F_{h}$ such that $N_{h} \rightarrow N$ and $D_{h} \rightarrow D$ in $M\left(\hat{\mathbf{A}}_{\sigma}\right)$.

Proof: Since all transfer functions in $M\left(\hat{\mathbf{B}}_{\sigma}\right)$ have left and right coprime factorizations [5], Vidyasagar's proof [21] for rational functions that convergence in the graph topology is equivalent to convergence of coprime factorizations extends in a straightforward manner to transfer functions in $M\left(\hat{\mathbf{B}}_{\sigma}\right)$.

The following result can be loosely paraphrased as follows: A family of plants $F_{h}$ can be robustly stabilized by a compensator $C$ which stabilizes some nominal plant $F$ if and only if $F_{h}$ converges to $F$ in the graph topology. Furthermore, the closed loop response of the feedback pair $\left(F_{h}, C\right)$ converges to that of $(F, C)$. In other words, the graph topology is the weakest topology in which feedback stability is robust.

Theorem 4.2: Let $F_{h}$ be a sequence of plants in $M\left(\hat{\mathbf{B}}_{\sigma}\right)$.

(i) Suppose $F_{h}$ converges to $F \in M\left(\hat{\mathbf{B}}_{\sigma}\right)$ in the graph topology. Let $C \in M\left(\hat{\mathbf{B}}_{\sigma}\right)$ stabilize $F$. Then there exists an $H$ such that $C$ stabilizes $F_{h}$, for all $h \leq H$, and moreover, the closed loop transfer matrix $H\left(F_{h}, C\right)$ converges to $H(F, C)$ in $M\left(\hat{\mathbf{A}}_{\sigma}\right)$.

(ii) Conversely, suppose there exists a $C \in M\left(\hat{\mathbf{B}}_{\sigma}\right)$ which stabilizes $F_{h}$ for all $h \leq H$, and that $H\left(F_{h}, C\right)$ converges to $H(F, C)$. Then $\left\{F_{h}\right\}$ converges to $F$ in the graph topology.

Proof: Identical to that in [21].

This has obvious implications for controller design using approximations. Failure of a sequence of approximations to converge in the graph topology implies that at least one of the following must occur (1) simultaneous stabilization of $F_{h}$ and $F$ is not possible for all $h$ sufficiently small or (2) convergence of the closed loop response $H\left(F_{h}, C\right)$ does not occur. 
Theorem 4.3: Let $(A, B, C)$ be a $\sigma$-stabilizable/detectable semigroup control system, and suppose we have a sequence of approximations $\left(A_{h}, B_{h}, C_{h}\right)$ satisfying assumptions (A1)(A3) of Section 2. If the system is $\sigma$-stabilizable and $C$ is any controller which stabilizes $P$, then for sufficiently small $H,\left(P_{h}, C\right)$ is $\sigma$-stable for all $h<H$. Furthermore, the closed loop response of $\left(P_{h}, C\right)$ converges in norm to that of $(P, C)$.

Proof: The theorem will be proven by using Theorem 3.3 to show that the approximate systems $\left(A_{h}, B_{h}, C_{h}\right)$ converge to $(A, B, C)$ in the graph topology, and then applying Theorem 4.2 .

Let feedback operators $K$ and $K_{h}$ be as defined above so that $A_{h o}:=A_{h}-B_{h} K_{h}$ generates a $\sigma$-stable semigroup $S_{h}(t)$ for sufficiently small $h$ and $A_{0}:=A-B K$ generates a $\sigma$-stable semigroup $S(t)$. For small $h$, right $\sigma$-coprime factorizations for the approximate systems are given by

$$
\begin{gathered}
\hat{N}_{h}(s)=C_{h} R\left(s: A_{h o}\right) B_{h}, \\
\hat{D}_{h}(s)=I-K_{h} R\left(s: A_{h o}\right) B_{h}
\end{gathered}
$$

where $R\left(s: A_{h o}\right)$ indicates the resolvent of $A_{h o}[21]$. A right coprime factorization for the original system is [13]

$$
\begin{gathered}
\hat{N}(s)=C R\left(s: A_{\circ}\right) B, \\
\hat{D}(s)=I-K R\left(s: A_{\circ}\right) B .
\end{gathered}
$$

The result follows if it can be shown that $N_{h}(t) \rightarrow N(t)$ and $D_{h}(t) \rightarrow D(t)$ in the norm on $\mathbf{A}_{\boldsymbol{\sigma}}$. Convergence of the numerators is proven first.

Since $\left\{\tilde{S}_{h}(t)\right\}$ converge uniformly on bounded intervals to $S(t)$, there exist $H$ and $M \geq 1$ such that for some $\gamma>\sigma$

$$
\|S(t)\| \leq M e^{-\gamma t} \text { for all } t \geq 0
$$

and

$$
\left\|\tilde{S}_{h}(t)\right\| \leq M e^{-\gamma t} \text { for all } t \geq 0, \quad \text { for all } h \leq H .
$$

Therefore, for any $\epsilon>0$ there exists $\tau$ such that

$$
\int_{\tau}^{\infty} \exp (\sigma t)\left\|N(t)-N_{h}(t)\right\| d t<\frac{\epsilon}{2}
$$

Also,

$$
\sup _{0 \leq t \leq \tau}\left\|N(t)-N_{h}(t)\right\| \leq \sup _{0 \leq t \leq \tau}\|C\|\left\|S(t) B-\tilde{S}_{h}(t) B\right\| .
$$

It follows from Theorem 3.3 that this also approaches zero. Combining statements (4.3) and (4.4) we see that

$$
\lim _{h \leq 0} \int_{0}^{\infty} \exp (\sigma t)\left\|N(t)-N_{h}(t)\right\| d t=0
$$


and so $N_{h}$ converges to $N$ on $\mathbf{A}_{\sigma}$.

The proof showing convergence of the denominators is somewhat lengthier and requires use of a lemma in [16]. Defining

$$
F_{h}(t):=\left(P_{h} \Pi-\Pi\right) S_{h}(t) P_{h} B
$$

we have

$$
\begin{aligned}
& D_{h}(t)-D(t)=Q^{-1} B^{*} F_{h} \overline{(t)}+Q^{-1} B^{*} \Pi\left[S_{h}(t) P_{h} B-S(t) B\right] . \\
& \lim _{h \rightarrow 0}\left\|\int_{0}^{\infty} e^{-\lambda t} F_{h}(t) d t\right\| \leq \lim _{h \rightarrow 0}\left\|\left(P_{h} \Pi-\Pi\right) \int_{0}^{\infty} e^{-\lambda t} \tilde{S}_{h}(t) B d t\right\| \\
& \leq \lim _{h \rightarrow 0}\left\|\left(P_{h} \Pi-\Pi\right) R\left(\lambda ; \tilde{A}_{h o}\right) B\right\| \\
& \leq \lim _{h \rightarrow 0}\left\|P_{h} \Pi\right\|\left(R\left(\lambda ; \tilde{A}_{h o}\right) B-R\left(\lambda_{;} A_{o}\right) B \|\right. \\
&+\left\|\left(P_{h}-I\right) \Pi R\left(\lambda ; A_{o}\right) B\right\| \\
&+\|\Pi\| \|\left(R\left(\lambda ; \tilde{A}_{o}\right) B-R\left(\lambda ; A_{h o}\right) B \|\right. \\
&=0,
\end{aligned}
$$

since $P_{h}$ converges strongly to the identity and

$$
\lim _{h \rightarrow 0}\left\|R\left(\lambda ; \tilde{A}_{h_{o}}\right) B-R\left(\lambda ; A_{o}\right) B\right\|=0
$$

for all $\lambda[18$, Theorem 4.2]. The sequence will now be shown to be equicontinuous .

The core $\mathrm{C}$ is dense in $\mathrm{D}(A)$ which is dense in $X$. Let $\left\{e_{i}\right\}_{i=1}^{n}$ be an orthonormal basis for $R^{m}$ and for any $\delta>0$ define a bounded operator $G_{\delta}: R^{m} \rightarrow X$ by

$$
G_{\delta}\left(e_{i}\right)=x_{i} \quad \text { where } \quad x_{i} \in \mathrm{C} \text { and }\left\|B e_{i}-x_{i}\right\|<\delta / m .
$$

Then $G_{\delta}$ is a bounded linear operator with range contained in $\mathbf{C}$ and with

$$
\left\|G_{\delta}-B\right\|<\delta
$$

Now $\left\|\tilde{A}_{h_{0}} G_{\delta}\right\|$ is bounded by some real $M_{1}$ since it converges to $\left\|A_{0} G_{\delta}\right\|$. Therefore,

$$
\begin{aligned}
\left\|F_{h}(t+\delta)-F_{h}(t)\right\| & \leq\left\|P_{h} \Pi-\Pi\right\|\left\|\int_{0}^{\delta} \tilde{S}_{h}(t+u) \tilde{A}_{h o} B d u\right\| \\
& \leq\|\Pi\|\left\|\int_{0}^{\delta} \tilde{S}_{h}(t+u) \tilde{A}_{h o} G_{\delta} d u\right\|+ \\
& \leq\|\Pi\|\left\|\left[\tilde{S}_{h}(t+\delta)-\tilde{S}_{h}(t)\right]\left(B-G_{\delta}\right)\right\| .
\end{aligned}
$$


Let $M$ be the constant bounding $S_{h}(t)$ for small $h$ (Equation 5.2 ). Then

$$
\left\|F_{h}(t+\delta)-F_{h}(t)\right\| \leq\|\Pi\|\left[\delta M M_{1}+2 M \delta\right]
$$

and for sufficiently small $H$ and any $\epsilon>0$ we can choose $\delta$ so that

$$
\left\|F_{h}(t+\delta)-F_{h}(t)\right\| \leq \epsilon \quad \text { for all } h<H
$$

Thus $F_{h}$ is an equicontinuous family and since $F_{h}(t)$ is also clearly uniformly bounded for all $t$ and $h$, it follows from [16, Lemma 2.11] that

$$
\lim _{h \rightarrow 0} \sup _{0 \leq t \leq \tau}\left\|F_{h}(t)-F(t)\right\|=0
$$

Since $\Pi$ and $Q^{-1} B^{*}$ are bounded operators it follows from Equation (4.5) and Theorem 3.3 that

$$
\lim _{h \rightarrow 0} \sup _{0 \leq t \leq \tau}\left\|D_{h}(t)-D(t)\right\|=0 .
$$

Finally, the remainder of the proof of convergence of $D_{h}(t)$ to $D(t)$ follows as for the numerators (4.3).

Convergence in the graph topology follows from Theorem 4.1. The conclusions of the theorem then follow from Theorem 4.2.

The result is independent of the technique used for stabilization, and does not require any assumptions on the stabilizability or detectability of the approximations. For instance, let $\left(N_{h}, D_{h}\right)$ be a $\sigma$-stable r.c.f. of an approximating plant and choose $X, Y \in M\left(\hat{\mathbf{A}}_{\sigma}\right)$ such that

$$
X N_{h}+Y D_{h}=I
$$

Then if the original plant is close enough to the approximation so that, for some r.c.f of the original plant, $(N, D)$,

$$
\left\|\begin{array}{l}
N-N_{h} \\
D-D_{h}
\end{array}\right\|<\frac{1}{\|X Y\|}
$$

then, any controller designed using the approximating plant will stabilize the original plant. Furthermore, the closed-loop responses will be close in norm, and the system will be internally $\sigma$-stable. The difficult part, of course, is determining whether (4.6) is satisfied.

\section{Conclusions}

The above results validate the use of Galerkin type approximations in controller design for infinite-dimensional systems, regardless of the control technique, and with very weak assumptions on the approximation method. The key assumption is that of stabilizability/ 
detectability of the semigroup control system which supports the work of Jacobson and Nett [14] indicating the importance of this property in control of infinite-dimensional systems.

A high order Galerkin model can be chosen and used to design a controller using lumped parameter techniques. If the objective is optimal state feedback, then convergence of the Kalman gains can be used as a criterion. Otherwise, robustness of the approximate plant with respect to the original infinite-dimensional system can be estimated before any controller is implemented by either (1) comparison of coprime factors (if available) or (2) determination that the sequence of approximations has converged satisfactorily. Several possible criteria are given in [21] and [11]. An open problem is determining when the approximation order is high enough for satisfactory controller design. Since exact transfer functions will be unavailable for most practical problems, a useful result would be the rate of convergence of the coprime factors, compared to that of the numerical scheme.

A subsequent paper will extend the results in this paper to discrete time approximation schemes.

\section{Acknowledgement}

Much of this material formed a part of my dissertation at the University of Waterloo and I would like to thank Prof. M. Vidyasagar for his guidance and encouragement during the development of these ideas. 


\section{References}

[1] Balas, M. J., Exponentially Stabilizing Finite-Dimensional Controllers for Linear Distributed Parameter Systems: Galerkin Approximation of Infinite Dimensional Controllers, J. Math. Anal. Appl., Vol. 117, 1986, pp. 358-384.

[2] Balas, M. J., Finite-Dimensional Control of Distributed Parameter Systems by Galerkin Approximation, J. Math. Anal. Appl., Vol. 114, 1986, pp. 17-36.

[3] Banks, H. T. and Kunisch, K., The Linear Regulator Problem for Parabolic Systems, SIAM J. Control Optim., Vol. 22, No. 5, 1984, pp. 684-698.

[4] Burns, John, Ito, Kazufumi, and Propst, Georg, On Non-Convergence of Adjoint Semigroups for Control Systems with Delays, ICASE Report No. 87-47, NASA Langley Research Center, July 1987.

[5] Callier, F. M. and Desoer, C. A., An Algebra of Transfer Functions of Linear Distributed Linear Time-Invariant Systems, IEEE Trans. Circuits and Systems, AC-25, Sept. 1978, pp. 651-662.

[6] Desoer, C. A. and Vidyasagar, M., Feedback Systems: Input-Output Properties, Academic Press, New York, 1975.

[7] Fairweather, Graeme, Finite Element Galerkin Methods for Differential Equations, Marcel Dekker, New York, 1978.

[8] Gibson, J. S., The Riccati Integral Equations for Optimal Control Problems on Hilbert Spaces, SIAM J. Control Optim., Vol. 17, 1979, pp. 537-565.

[9] Gibson, J. S., Linear-Quadratic Optimal Control of Hereditary Differential Systems: Infinite Dimensional Riccati Equations and Numerical Approximations, SIAM J. Control Optim., Vol. 21, 1983, pp. 95-139.

[10] Gibson, J. S. and Rosen, I. G., Numerical Approximation for the Infinite-Dimensional Discrete-Time Optimal Linear-Quadratic Regulator Problem, SIAM J. Control Optim., Vol. 26, 1979, pp. 428-451.

[11] Glover, K. and McFarlane, D., Robust Stabilization of Normalized Coprime Factor Plant Descriptions with $H_{\infty}$-Bounded Uncertainty, IEEE Trans. Circuits and Systems, AC-34, August 1989, pp. 822-830. 
[12] Ito, Kazufumi, Strong Convergence and Convergence Rates of Approximating Solutions for Algebraic Riccati Equations in Hilbert Spaces, Proceedings of the 3rd International Conference on Distributed Parameter Systems, Springer-Verlag, Berlin, 1986.

[13] Jacobson, Clas A., "The Structure of Exponential Stabilization of a Class of Linear Distributed Parameter Systems", Ph.D. Thesis, Rensselaer Polytechnic Institute, Troy, NY, 1986.

[14] Jacobson, Clas. A. and Nett, Carl N., Linear State-Space Systems in InfiniteDimensional Space: The Role and Characterization of Joint Stabilizability/Detectability, IEEE Trans. Automat. Control, BAC-33, June 1988, pp. 541-549.

[15] Kato, T., Perturbation Theory for Linear Operators, Springer-Verlag, 1966.

[16] Kurtz, Thomas G., Extensions of Trotter's Operator Semigroup Approximation Theorems, J. Funct. Anal., Vol. 3, 1969, pp. 354-375.

[17] Lukes, D. L. and Russell, D. L., The Quadratic Criterion for Distributed Systems, SIAM J. Control, Vol. 7, No. 1, 1969, pp. 101-121.

[18] Pazy, A., Semigroups of Linear Operators and Applications to Partial Differential Equations, Springer-Verlag Inc., 1983.

[19] Rosen, I. G., On Hilbert-Schmidt Norm Convergence of Galerkin Approximation for Operator Riccati Equations, NASA Contractor Report 181755, ICASE Report No. 8871, November 1988.

[20] Strang, Gilbert, Approximating Semigroups and the Consistency of Difference Schemes, Proc. Amer. Math. Soc., Vol. 20, 1969, pp. 1-7.

[21] Vidyasagar, M., Control System Synthesis: A Factorization Approach, MIT Press, Cambridge, 1985 .

[22] Vidyasagar, M., The Graph Metric for Unstable Plants and Robustness Estimates for Feedback Stability, IEEE Trans. Automat. Control, Vol. AC-29, No. 5, May 1984.

[23] Weidmann, Joachim, Linear Operators in Hilbert Spaces, Springer-Verlag, 1980.

[24] Zames, G. and El-Sakkary, A., Unstable Systems and Feedback: The Gap Metric, Proc. Allerton Conf., 1980, pp. 380-385.

[25] Zhu, S. Q., Graph Topology and Gap Topology for Unstable Systems, IEEE Trans. Automat. Control, Vol. AC-34, No. 8, August 1989, pp. 848-855. 





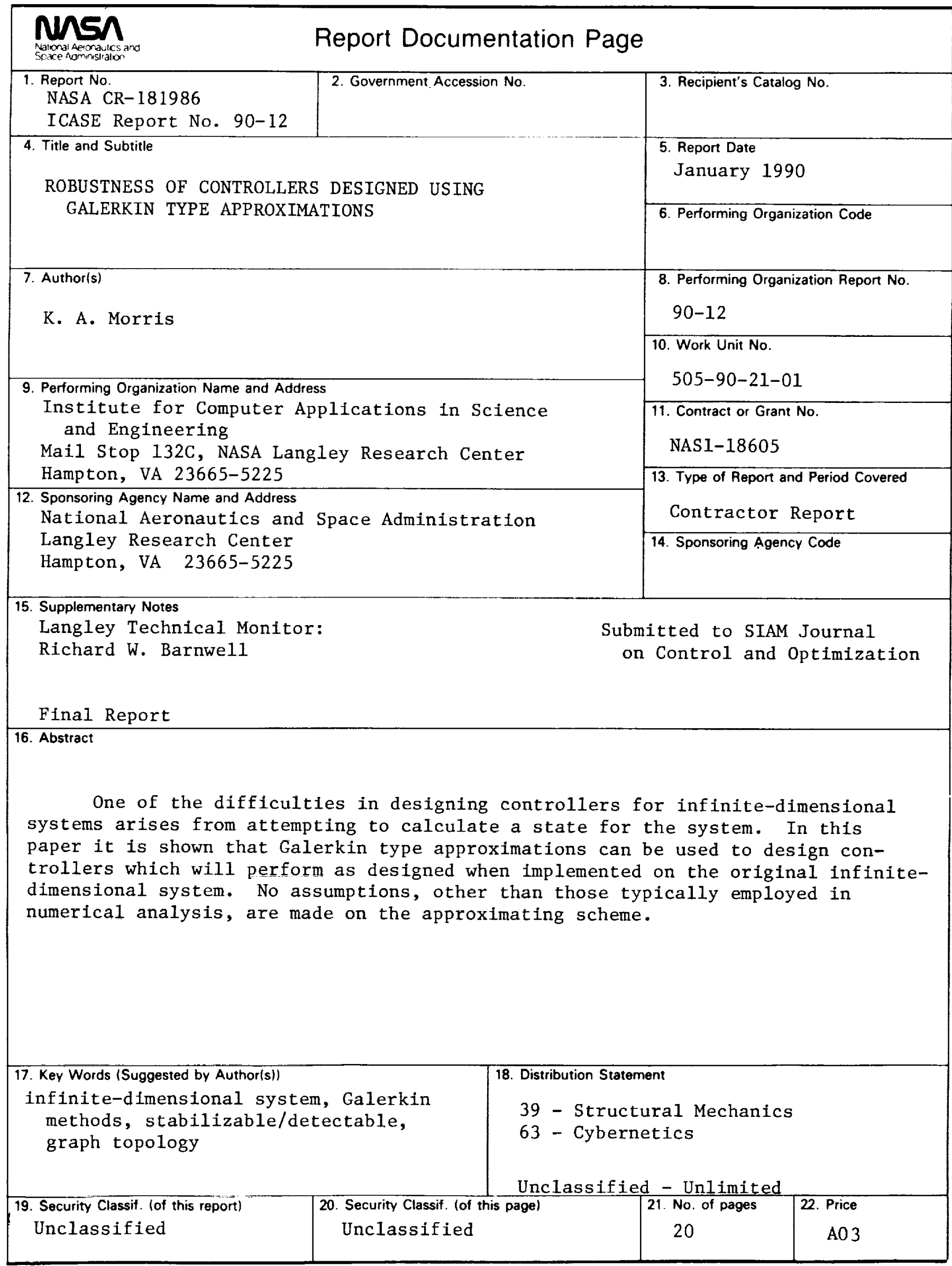

